\title{
Locke, Arbeit und Emanzipation
}

\author{
Von ULRICH STEINVORTH (Ankara)
}

Arbeit, labour, war für Locke der Weg zur Emanzipation des Menschen, seiner Befreiung aus Abhängigkeiten, die seinen Fähigkeiten unangemessen sind. Arbeit war ihm auch das Mittel zur Vervollkommnung, wenn nicht zur Vollkommenheit des Menschen. Viele seiner Annahmen zur Arbeit widersprechen heutigen Kenntnissen und Vorstellungen, aber in einer zentralen These hat er nicht nur Rousseau, Kant, Hegel und Marx beeinflusst; sie ist auch richtig, wie ich zeigen möchte. Es ist die These, dass für alle Menschen durch eine bestimmte Form der Wirtschaft oder des Einsatzes verfügbarer Ressourcen ein aktiver Gebrauch ihrer rationalen Fähigkeiten möglich ist, der ihnen das bringt, was Philosophen seit Platon von solchem Gebrauch erwartet haben: Glück, Freiheit, Vollkommenheit.

Die radikale Neuerung, die Locke der Philosophie vermacht hat, ist weder, dass die Menge der Arbeit, die in ein Produkt eingeht, ihren Wert bestimmt, noch dass die Arbeit die unerschöpfliche Quelle des Reichtums ist, die den Mangel natürlicher Ressourcen ausgleichen kann. Diese Thesen Lockes sind zwar wichtig genug, um ihm einen Platz in der Ideengeschichte zu sichern. Aber sie sind irrig und nicht zentral für Lockes Revolution. Die besteht darin, das antike Ideal der Vernunfttätigkeit universalisiert, historisiert und politisiert zu haben. Locke zeigte, wie alle Menschen durch eigene ökonomisch relevante Tätigkeit die oft zitierte, aber selten befolgte Idee des Vernunftgebrauchs verwirklichen können. Er verfocht bürgerliche Interessen, aber war auch der erste Marxist, der Marx' These vom bestimmenden Einfluss der Wirtschaft auf Politik und Moral vorwegnahm und doch so wenig wie Marx zum moralischen Relativisten wurde. Denn auch er hielt an einem Vernunftideal fest, das Selbsttätigkeit zur notwendigen und hinreichenden Bedingung menschlicher Vollkommenheit macht.

Ich beginne mit der Darlegung der zwei erwähnten Thesen, durch die Locke als Verfechter der so genannten Arbeitswerttheorie bekannt wurde, der These:

(A) Der ökonomische Wert eines Produkts ist proportional der Menge der Arbeit, die in es eingegangen ist,

und der These:

(R) Arbeit und nicht natürliche Ressourcen sind die Quelle des Reichtums eines Landes.

Lockes eigene Argumente für A und R werden zeigen, dass er sie als Stützen der These betrachtete, die vermutlich ihm selbst, in jedem Fall aber für das heutige Erkenntnisinteresse am wichtigsten ist, der These: 
(L) Wir leben in ökonomischen Bedingungen, die jedem erlauben, seine rationalen Fähigkeiten ohne Anleitung durch andere selbst zu betätigen.

\section{Arbeit als der Weg zur Vollkommenheit}

Locke argumentiert für A und R, um Eigentum, die Aneignung natürlicher Ressourcen zu rechtfertigen. Ursprünglich und grundsätzlich gilt:

„The Earth, and all that is therein [...] belong[s] to Mankind in common, as they are produced by the spontaneous hand of Nature; and no body has original a private Dominion, exclusive of the rest of Mankind, in any of them. ${ }^{\text {"1 }}$

In dieser Annahme stimmt Locke mit dem Naturrecht der Scholastik und Ciceros überein. ${ }^{2}$ Abgesehen von so wichtigen Dingen wie Wasser und Luft sind die meisten Gebrauchsdinge, Häuser, Töpfe, Äpfel, jedoch Produkte aus Natur und menschlicher Arbeit. Wir brauchen für solche Dinge einerseits natürliche Materialien und vorgefundene natürliche Arten, aus denen Häuser und Töpfe gemacht und Apfelbäume gezüchtet wurden, und andererseits die Arbeit der Haus- und Topfmacher und Apfelbauern. Weil Marx eine ähnliche Arbeitswerttheorie wie Locke zu vertreten schien, erklärte bei ihrer Gründung 1875 die Sozialistische Arbeiterpartei Deutschlands (SAPD, 1890 in Sozialdemokratische Partei Deutschlands, SPD, umbenannt) in ihrem Gothaer Programms:

„Die Arbeit ist die Quelle alles Reichtums und aller Kultur.“

Marx widersprach bekanntlich:

„Die Arbeit ist nicht die Quelle alles Reichtums. Die Natur ist ebensosehr die Quelle der Gebrauchswerte (und aus solchen besteht doch wohl der sachliche Reichtum!) als die Arbeit, die selbst nur die Äußerung einer Naturkraft ist, der menschlichen Arbeitskraft. [...] Die Bürger haben sehr gute Gründe, der Arbeit übernatürliche Schöpfungskraft anzudichten; denn grade aus der Naturbedingtheit der Arbeit folgt, daß der Mensch, der kein andres Eigentum besitzt als seine Arbeitskraft, in allen Gesellschafts- und Kulturzuständen der Sklave der andern Menschen sein muß, die sich zu Eigentümern der gegenständlichen Arbeitsbedingungen gemacht haben. Er kann nur mit ihrer Erlaubnis arbeiten, also nur mit ihrer Erlaubnis leben."3

Aber auch Locke leugnet nicht den Anteil der Natur; er schätzt ihn nur als gering ein:

1 J. Locke, Two Treatises of Government, hg. v. P. Laslett, Cambridge 1960, II, § 26. Locke nehme ursprüngliches Gemeineigentum aus theologischen Gründen an, wird oft behauptet. Tatsächlich beruft er sich hier wie in vielen anderen Argumenten zugleich auf ,natural Reason" und „Revelation“; wie das Zitat zeigen kann, ist das Vernunftargument für Locke wichtiger: Weil die Natur und kein Mensch eine natürliche Ressource hervorbringt, hat kein Mensch ein Eigentumsrecht auf sie, solange niemand durch Arbeit Wert schafft.

2 Vgl. Cicero, De officiis, hg. v. H. Gunermann, Stuttgart 1984, I, 51 u. 23. Cicero verteidigt Privateigentum wie Locke, aber begründet es nicht durch ein natürliches Recht auf Aneignung durch Arbeit, sondern allein durch den Nutzen für die Gesellschaft.

3 K. Marx, Kritik des Gothaer Programms, in: Marx-Engels-Werke (MEW), Bd. 19, 15. 
„t'is Labour indeed that puts the difference of value on every thing; and let any one consider, what the difference is between an Acre of Land planted with Tobacco, or Sugar, sown with Wheat or Barley; and an Acre of the same Land lying in common, without any Husbandry upon it, and he will find, that the improvement of labour makes the far greater part of the value. I think it will be but a very modest Computation to say, that of the Products of the Earth useful to Life of Man 9/10 are the effects of labour: nay, if we will rightly estimate things as they come to our use, and cast up the several Expences about them, what in them is purely owing to Nature, and what to labour, we shall find, that in most of them 99/100 are wholly to be put on the account of labour. ${ }^{64}$

Drei Paragraphen weiter erklärt Locke den Anteil der Natur am Produktwert sogar, „I may truly say, not $1 / 1000$ “. 5

Lockes Argument ist irrig, und daher sind A und R falsch. Denn es ist der Grad der Knappheit, die den Marktwert einer natürlichen Ressource ebenso wie den einer Arbeit bestimmt. Um nur ein Beispiel aus unzähligen zu nennen: Seit Lithium der Kandidat für den Stoff ist, aus dem Autobatterien gemacht werden sollen, ist sein Preis hochgeschossen, und Bolivien als Hauptquelle sieht sich der Begierde der Autoproduzenten ausgesetzt. ${ }^{6}$

Locke hätten jedoch vielleicht solche Beispiele nicht beeindruckt. Er könnte darauf bestanden haben, Knappheit bestimme den Preis, nicht den Wert. Nur unter Marktbedingungen spiele Knappheit eine Rolle, ihm aber gehe es um eine Analyse, die auf jede Wirtschaft zutrifft, nicht nur die Marktwirtschaft. Diese Verteidigung ist zwar unhaltbar, da Locke Geld als Wertmaßstab annimmt ${ }^{7}$ und Geld Markttausch voraussetzt. Sie entspricht jedoch Lockes Absicht, die Arbeit als den Faktor auszuzeichnen, dessen Menge und Qualität anders als die natürlicher Ressourcen vom Willen oder der „Person“ jedes Individuums abhängt.

Die „Person“, so erklärt Locke, ist anders als die natürlichen Ressourcen etwas, was ursprünglich privates Eigentum eines jeden ist:

„[...] every Man has a Property in his own Person. This no Body has any Right to but himself. The Labour of his Body, and the Work of his Hands, we may say, are properly his." ${ }^{\text {" }}$

„Person“ wird oft als das körperliche entscheidungsfähige Individuum verstanden. Locke unterscheidet jedoch person vom Individuum, das er getreu seiner Arbeitswerttheorie als Eigentum seines Produzenten versteht, nicht der Eltern, sondern des „one Omnipotent, and infinitely wise Maker", der auch die Eltern produziert hat. Weil ich nicht Eigentum meiner selbst bin, darf ich nach Locke mein Leben nicht willkürlich beenden. „Men“ sind Gottes Eigentum. ${ }^{9}$ Meine Person dagegen ist die Eigenschaft, die meine Entscheidungen rechtsfähig macht und die ich verliere, wenn ich für unmündig erklärt oder versklavt werde. Daher hat „every Man [...] a Property in his own Person" ${ }^{\prime 10}$ und ist „Proprietor of his own Person“. ${ }^{11}$

4 J. Locke, Two Treatises, a. a. O., II, $\S 40$.

5 Ebd., $\S 43$.

6 Time, 22.1.2009.

7 J. Locke, Two Treatises, a. a. O., II, § 37.

8 Ebd., $\$ 27$.

9 Ebd., § 6.

10 Ebd., $\$ 27$.

11 Ebd., § 44. 
Von der person hängt es ab, ob und wie man arbeitet. Seine Arbeit aber ist nicht nur Eigentum jedes Individuums, von dessen Gebrauch es andere auszuschließen das Recht hat; sie ist auch die Kraft, die es einsetzen kann, um sich Reichtum und allgemeiner die Lebensumstände zu verschaffen, in denen es leben will.

Locke klärt nicht, wie die Eigenschaft eines Individuums A, das Gottes Eigentum ist, doch A's Privateigentum sein kann. Aber es lassen sich durchaus juristische und theologische Konstruktionen für einen Bereich privater Verantwortlichkeit auch eines Sklaven denken.

In jedem Fall machen Lockes Aussagen zum Eigentum klar, dass seine Theorien des Arbeitswerts und des Eigentums in eine Theorie der Verantwortlichkeit eingebettet sind, die das ursprünglich einzige Privateigentum, die eigene Person oder Entscheidungsfähigkeit, zur Quelle zugleich des Rechts auf private Aneignung natürlicher Ressourcen und der Pflicht macht, die Person zum Einsatz ihrer Arbeit zu gebrauchen. Da jeder Verantwortliche über die Quelle nicht nur der Verantwortlichkeit, sondern auch der Arbeit verfügt, findet er in seiner Arbeitskraft genügend Ressourcen vor, alles zu erreichen und zu erwerben, was ein Mensch erreichen kann.

Lockes berühmte Aneignungsbedingung, nach der jeder natürliche Ressourcen aneignen darf, auf die er hinreichende Arbeit verwendet hat, ,at least where there is enough, and as good left in common for others" ${ }^{\text {"12 }}$, erweist sich daher als leicht erfüllbar. Locke kann darauf setzen, dass der findige Arbeitswillige schon irgendwelche Naturgüter findet, auf die er seine Arbeit wendet. Wer weniger erfindungsreich ist, kann immer noch seine Arbeitskraft an den Findigeren verkaufen, kann aber dann als Produkt seiner Arbeit nur den vertraglich vereinbarten Lohn einstreichen, während sein Arbeitsprodukt an den Arbeitgeber fällt:

„[...] the Turfs my Servant has cut $[\ldots]$ become my Property. “13

Arbeitswilligkeit und Erfindungsgeist sind Bedingung für ökonomischen und sonstigen Erfolg, wie Locke hervorhebt:

„God gave the World to Men in Common; but since he gave it them for their benefit [...] it cannot be supposed he meant it should always remain common and uncultivated. He gave it to the use of the Industrious and Rational, (and Labour was to be his Title to it); not to the Fancy and Covetousness of the Quarrelsom and Contentious." ${ }^{14}$

In heutigen Ohren klingen solche Aussagen abstoßend selbstgerecht. Im 17. Jahrhundert lag ihre Bedeutung in der Kritik feudaler Politik. Die „Quarrelsom and Contentious“ ist nicht die Klientel, die heutige Liberale wie Westerwelle in Hartz IV-Empfängern sehen; es ist die Aristokratie, gegen deren ,vainglory“ schon Hobbes bürgerliche Rationalität setzte. ${ }^{15}$

Locke erkannte ebenso klar wie Marx den Ursprung bürgerlicher Rationalität im Gebrauch von Geld, der den Tausch verderblicher Produkte gegen unvergängliche Edelmetalle ermöglichte ${ }^{16}$, und sah ihre Schattenseiten: die aus dem Interesse an profitablem Markttausch folgenden ,vain Ambition, and amor sceleratus habendi, evil Concupiscence“. ${ }^{17}$

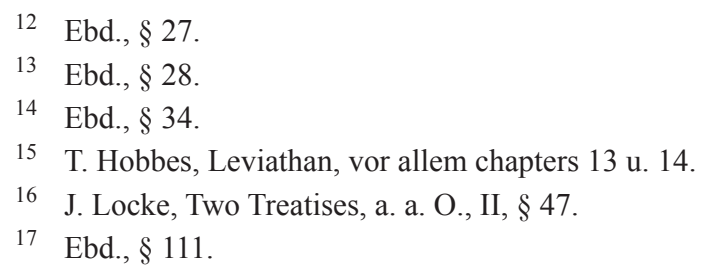


„Find out something that hath the Use and Value of Money amongst his Neighbours, you shall see the same Man will begin presently to enlarge his Possessions." 18

Anders aber als Marx und sicher weniger realistisch sieht Locke die Besitzgier langfristig realisierbar nur dann, wenn sie zu einer Bearbeitung des Bodens führt. Diese verbessert einerseits die Natur im selben Maß, wie der Anteil der Arbeit am Wert des Produkts den Anteil der Natur übertrifft, nämlich um mehr als das Tausendfache. ${ }^{19}$ Anderseits vervollkommnet sie auch die rohe menschliche Natur, da die Kultivierung des Bodens und anderer natürlicher Ressourcen mit einer Kultivierung der rationalen Fähigkeiten der Menschen zusammengeht. Locke müht sich, seine Idee des Zugleichs von Gier, Werterhöhung der Natur durch Arbeit und Kultivierung menschlicher Fähigkeiten quantitativ darzustellen:

„[...] let us but trace some of the ordinary provisions of Life, through their several progresses, before they come to our use, and see how much they receive of their value from Humane Industry. Bread, Wine and Cloth, are things of daily use, and great plenty, yet notwithstanding, Acorns, Water, and Leaves, or Skins, must be our Bread, Drink, and Clothing, did not labour furnish us with these more useful Commodities. " 20

Während seine quantitative These vom mehr als tausendfachen Wert der Arbeit über der Natur fehlgehen muss, ist seine implizite qualitative These der Entwicklung rationaler Fähigkeiten durch die Kultivierung der Natur in „Humane Industry“ richtig. Was diese These in heutiger Perspektive besonders interessant macht, aber auch leicht an ihr übersehen werden kann, ist die Annahme, die Entwicklung rationaler Fähigkeiten durch Arbeit oder in Bearbeitung der Natur sei spontan oder selbsttätig, weil die menschliche Natur zu ihr neigt und keiner Anleitung durch Staat oder Verwaltung bedarf:

„[...] numbers of men are to be preferd to largenesse of dominions, and [...] the increase of lands and the right imploying of them is the great art of government. And that Prince who shall be so wise and godlike as by established laws of liberty to secure protection and incouragement to the honest industry of Mankind against the oppression of power and narrownesse of Party will quickly be too hard for his neighbours. “21

Die beste Regierung, so Lockes Voraussetzung nicht nur in seinen Erklärungen zu den Zielen der Staatsgründung, ist die, die die Freiheit der Bürger in der Bearbeitung natürlicher Ressourcen schützt und ihrer Natur zur Betätigung ihrer rationalen Fähigkeiten freien Lauf lässt. Dieser Gedanke ist heute eng mit der Idee der Marktfreiheit verbunden und ebenso in Verruf geraten wie die Idee der Marktfreiheit selbst. Aber Locke denkt die Freiheit für die Betätigung rationaler Fähigkeiten nicht von vornherein als Marktfreiheit, sondern als Freiheit, die eigenen rationalen Fähigkeiten zu betätigen. Der gottgleiche Fürst lässt seinen Bürgern die Freiheit, ihre Fähigkeiten an Naturgütern wie dem Land zu betätigen. Da Arbeit und nicht Land die Quelle des Reichtums ist, wird er Menschen und nicht Land vermehren wollen. Fördert der Markt die Entwicklung rationaler Fähigkeiten, wird der Fürst den Markt schützen. Aber sein erstes Interesse ist zu verhindern, dass natürliche Ressourcen und menschliche

\footnotetext{
18 Ebd., $§ 49$.

19 Ebd., $\S 43$, cp. $\S \S 37$ u. 40.

20 Ebd., $\$ 42$.

21 Ebd., § 42.
} 
Fähigkeiten brachliegen. ${ }^{22}$ Ein Marktsystem, das wie das heutige Arbeitslosigkeit fördert, ist unvereinbar mit Lockes Idee des gottgleichen Fürsten.

Ein Musterbeispiel für Lockes Annahme der Selbsttätigkeit der Entwicklung rationaler Fähigkeiten, aber auch ihrer Verflechtung mit moralischer Unvollkommenheit, ist seine Erklärung dessen, was er die „Invention of Money“ nennt. ${ }^{23}$ Sie hat zwei natürliche Quellen. Einerseits „Fancy or Agreement“, womit Menschen unverderblichen und hübschen Dingen wie „Gold, Silver, and Diamonds“ einen Wert verliehen haben, der größer ist als ihr ,real Use““. ${ }^{24}$ Anderseits ihre Rolle im Tausch gegen die verderblichen Güter, die aus der Bearbeitung natürlicher Ressourcen entstehen. Nur wenn diese Produkte austauschbar gegen haltbare Dinge sind, vermehrbar ohne Gefahr zu verderben, werden Menschen mehr produzieren als für ein bequemes Leben nötig ist und dadurch auch ihre rationalen Fähigkeiten über das Maß des Nötigen hinaus entwickeln. Nur wenn sie Aussicht auf solchen Tausch haben, gibt es einen ,place of Money“:

„What would a Man value Ten Thousand, or an Hundred Thousand Acres of excellent Land, ready cultivated, and well stocked with Cattle, in the middle of the in-land Parts of America, where he had no hopes of Commerce with other Parts of the World, to draw Money to him by Sale of the Product? It would not be worth the inclosing, and we should see him give up again to the wild Common of Nature, whatever was more than would supply the Conveniences of Life to be had there for him and his Family. “25

Ihre Natur macht die Menschen zugleich anfällig für das Sammeln schöner und haltbarer Dinge und für die Betätigung ihrer Anlagen; der Tausch von Arbeitsprodukten gegen hübsche Dinge macht aus diesen zugleich Maß und Speicher von Wert. Zwar müssen auch Tauschbedingungen gegeben sein, um die Entwicklung auszulösen, die schließlich mit der Verbesserung der Natur auch zur Verbesserung der Menschen führt. Aber diese Bedingungen waren in Europa und Asien gegeben; daher entwickelten sich Handel und mit ihm die Verbesserung der Natur und der Menschen. Jeder Eingriff in diesen Prozess der Selbsttätigkeit, der die Menschen an der natürlichen Entfaltung ihrer Anlagen hinderte, ist schädlich. ${ }^{26}$

22 Ebd., $\S 42 ;$ cp. $\S \S 38,45$.

23 Ebd., $\S \S 36,48$.

24 Ebd., § 46. Locke unterscheidet hier Gebrauchs- und Tauschwert.

25 Ebd., § 48. Inclosing ist die Einzäunung von Land, das zuvor Allmende oder Gemeineigentum war. Rousseau geißelte in seinem Discours über die Ungleichheit die erste Einzäunung von Land als den großen Betrug, der „la société civile“ und „que de crimes de guerres, de meurtres, que de misères et d'horreurs“" einleitete (J.-J. Rousseau, Schriften zur Kulturkritik, Hamburg 1971, 190 f.). Lockes Rechtfertigung privater Aneignung nimmt das heutige Argument der "tragedy of the commons" vorweg. Garrett Hardin (The Tragedy of the Commons, in: Science, 162 (1968), 1243-1248) verwies wie viele vor ihm auf die Gefahr der Übernutzung von Allmenden. Carol M. Rose (The Comedy of the Commons: Commerce, Custom and Inherently Public Property, in: University of Chicago Law Review, 711 (1986), 53) wies auf den Nutzen von Allmenden. Michael A. Heller (The Tragedy of the Anticommons, in: Harvard Law Review, 111 (1998)) und M. A. Heller sowie Rebecca Eisenberg (Can Patents Deter Innovation? The Anticommons in Biomedical Research, in: Science, 280 (1998)) zeigten die Gefahr der Unternutzung von Gütern durch Patente (vgl. allgemeiner: Wem gehört die Welt?, hg. v. S. Helfrich, München 2009).

26 Die perfektionistische Interpretation des Liberalismus im Allgemeinen und Lockes im Besonderen ist beeinflusst von: V. Haksar, Equality, Liberty, and Perfectionism, Oxford 1979; vgl. E. Riley, Locke's Perfectionist Liberalism: An Articulation and Defense. Doctoral Dissertation, University of Pittsburgh, 2008. Zentral für die perfektionistische Interpretation scheint mir Lockes Aussage 
Wir müssen am Geschichtsbild, das Locke in seiner Beschreibung der Entwicklung von Eigentum und Geld im Naturstand entwirft, zwei gleichwichtige Aspekte unterscheiden. Der erste und bekanntere ist das Motiv der felix culpa, das oft erst späteren Autoren zugeschrieben wird. Aber schon nach Locke vertreibt der Prozess der Vervollkommnung natürlicher Ressourcen und rationaler Fähigkeiten die Menschen aus dem „Golden Age (before vain Ambition, and amor sceleratus habendi, evil Concupiscence, had corrupted Mens minds into Mistake of true Power and Honour) “. ${ }^{27}$ Die Vertreibung aus dem Paradies ist Folge des Sündenfalls des Geldgebrauchs. Für Locke wie für Marx ist er eine felix culpa, da die rationalen und initiativen Fähigkeiten der Menschen ohne den Sündenfall unentwickelt geblieben wären. Oder wie Kant denselben Gedanken ausdrückte:

\begin{abstract}
„Das Mittel, dessen sich die Natur bedient, die Entwickelung aller ihrer Anlagen zustande zu bringen, ist der Antagonism derselben in der Gesellschaft [...] die ungesellige Geselligkeit der Menschen [...] Ohne jene, an sich zwar eben nicht liebenswürdige Eigenschaften der Ungeselligkeit [...], würden in einem arkadischen Schäferleben, bei vollkommener Eintracht, Genügsamkeit und Wechselliebe, alle Talente auf ewig in ihren Keimen verborgen bleiben; die Menschen, gutartig wie die Schafe, die sie weiden, würden ihrem Dasein kaum einen größeren Wert verschaffen als dieses ihr Hausvieh hat; sie würden das Leere der Schöpfung in Ansehung ihres Zwecks als vernünftige Natur nicht ausfüllen." ${ }^{28}$
\end{abstract}

Die Menschen, so der übereinstimmende Gedanke von Locke über Kant und Hegel zu Marx, haben rationale Fähigkeiten, die sie vor den Tieren auszeichnen, aber höchstens in Einzelfällen betätigen, wenn sie nicht eine bestimmte Gesellschaftsform zu ihrer Betätigung zwänge. Es gäbe, wie Kant formuliert, eine „Leere der Schöpfung“, ein missing link in der Stufenleiter des Seins von der göttlichen Vernunft zur Vernunft- und Leblosigkeit der niedersten Ränge der Schöpfung ${ }^{29}$, wenn die Menschen von der Möglichkeit der Betätigung ihrer fast göttlichen Vernunft nicht Gebrauch machten.

Der zweite und revolutionäre Aspekt in Lockes Geschichtsentwurf, von dem Kants Rückgriff auf die metaphysische Annahme einer Leiter des Seins leicht ablenken kann, ist, dass die Vervollkommnung der Menschen und der Natur durch die Selbsttätigkeit der Individuen erfolgt. Für Kant ist die Idee der Vervollkommnung durch die Laster von Gier und Herrschsucht ein Grund, trotz einer ,,aus Torheit, kindischer Eitelkeit, oft auch aus kindischer Bosheit und Zerstörungssucht zusammengewebten" Geschichte ${ }^{30}$ für die Zukunft zu hoffen. Für Locke ist sie ein Grund, die Eigeninitiative und Spontaneität der Individuen hervorzuheben, die zwar die Schattenseite der Gier hat, aber in ihrem Austragen der guten Frucht nicht gestört werden darf durch die „oppression of power and narrownesse of Party“, zu denen Regierungen neigen. ${ }^{31}$

in $\S 33$ des Zweiten Treatise, Gott habe die Welt zum Gebrauch der „Industrious and Rational“ gegeben.

27 J. Locke, Two Treatises, a. a. O., II, § 111.

28 I. Kant, Idee zu einer allgemeinen Geschichte, in: Kants Werke, hg. v. E. Casirer, B. 4, Berlin 1913, hier: Vierter Satz.

29 Vgl. dazu A. Lovejoy, The Great Chain of Being: A Study of the History of an Idea [1933], Cambridge/Mass. 2001; und U. Steinvorth, Warum überhaupt etwas ist. Kleine demiurgische Metaphysik, Reinbek 1994.

30 I. Kant, Idee zu einer allgemeinen Geschichte, a. a. O., Einleitung; vgl. den Neunten Satz.

31 J. Locke, Two Treatises, a. a. O., II, § 42. 
Lockes Absicht, die Aufgaben der Regierung auf die des Schutzes der Rechte der Individuen zu beschränken und diese Rechte vor allem als Eigentumsrechte zu verstehen, geht zusammen mit der Absicht, die Überlegenheit privaten Eigentums vor Gemeineigentum zu zeigen. Er beschreibt die private Aneignung als einen Vorgang im Naturstand, in dem es noch keine Regierung, daher auch keine feudalen oder anderen Herrschaftsverhältnisse gibt, die das ursprüngliche Privateigentum, die person, einschränken könnten. Eine Regierung wird erst wünschenswert, wenn nach Einführung des Geldes die ökonomische Ungleichheit so groß wird, dass die Armen begehrlich werden und die Besitzenden ihren Besitz, nach Locke ihr legitimes Eigentum, wenn durch ihre Arbeit erworben, durch die Einführung eines Systems der Legislative, Judikative und Exekutive schützen; eines Systems, dessen ,great and chief end“ Locke als ,the Preservation of their Property“ beschreibt. ${ }^{32}$

Locke erklärt zwar, er verstehe unter Property ,their Lives, Liberties and Estates“633; er versichert auch, die Gesetze des vertraglich vereinbarten Staates seien „one Rule for Rich and Poor, for the Favourite at Court, and the Country Man at Plough". ${ }^{34}$ Aber er hat keinen guten Grund dafür, warum ein Besitzloser, der im Naturstand nichts zu verlieren hat, sich einer Staatsgewalt unterwerfen und seine geliebte Freiheit aufgeben sollte. ${ }^{35}$ Und selbst wenn er das täte, würde er doch nicht zum Staatsbürger. Denn wie Locke erklärt,

„[...] submitting to the Laws of any Country, living quietly, and enjoying Priviledges and Protection under them, makes not a Man a Member of that Society [...] Nothing can make any Man so, but his actually entering into it by positive Engagement, and express Promise and Compact. “36

Als positive Engagement oder express Promise erkennt Locke an, dass ein Besitzender im Land bleibt ${ }^{37}$; vermutlich auch den Fahneneid eines Offiziers und Soldaten. Aber für Besitzlose ist nichts vorgesehen, wodurch sie Staatsbürger werden könnten. Locke hätte kaum klarer machen können, dass er wie Marx den Staat für eine Institution der herrschenden Klassen hält und sich von ihm nur durch die positive Bewertung der Staatsaufgabe des Eigentumsschutzes unterscheidet.

Seine positive Bewertung aber ist folgerichtig, weil er im Besitzerwerb den Weg zum Vernunftgebrauch und diesen Weg offen für jeden sieht. Dass er jedem offen steht, hält er nicht für eine Naturtatsache, auch wenn er diesen Punkt nicht anspricht. Vielmehr war es im 17. Jahrhundert klar genug, dass die Staatsgewalt die freie Verfügung der Individuen über sich - ihre person - einschränken könnte und würde, wenn die Bürger sie nicht daran hinderten, wie es in der Glorious Revolution von 1688 tatsächlich geschah. Der Staat ist für Locke die Institution des Eigentumsschutzes unter der Bedingung, dass jeder Besitz erwerben und dabei seine rationalen Fähigkeiten betätigen kann. Er ist der Weg zur Vervollkommnung aller Menschen, wenn nur alle Menschen frei zur Betätigung ihrer Arbeitskraft und damit des Vermögens sind, in dem jeder seine Vernunftfähigkeit entwickeln kann.

Diese Auffassung von Staat, Eigentum und Produktions- und Handelsfreiheit entsprach dem Interesse des Bürgertums und entspricht ihm noch heute, soweit es ein Bürgertum in der

\footnotetext{
32 Ebd., § 124.

33 Ebd., § 123.

34 Ebd., § 142.

35 Ebd., $§ 123$.

36 Ebd., § 122.

37 Ebd., § 119.
} 
Rolle, die es im 17. Jahrhundert hatte, noch gibt. Aber die Auffassung hat Bedeutung auch unabhängig von bürgerlichen Interessen. Sie besteht darin, die Entwicklung der rationalen Fähigkeiten als eine Tätigkeit zu sehen, die von den Individuen selbst ausgeübt werden kann und muss und von jedem obrigkeitlichen Eingriff gestört wird. Wir sehen diese Idee heute skeptisch, weil die liberalen Anhänger Lockes die Selbsttätigkeit zunehmend als Markttätigkeit verstanden haben und der Markt sich nicht als gleich förderlich für alle erwiesen hat. Für Locke war dagegen die Selbsttätigkeit zuerst die Arbeit, die Anwendung der Arbeitskraft auf natürliche Ressourcen. Dass diese Tätigkeit tatsächlich nur unter Marktbedingungen möglich und dadurch den Problemen einer Marktwirtschaft ausgesetzt war, wurde erst nach Locke deutlich.

Lockes zentrale Idee, dass eine Vervollkommnung der Individuen, ihre Emanzipation von den Behinderungen ihrer Fähigkeiten, nur durch eigene Tätigkeit möglich ist, teilte auch Marx. Den Problemen der Markttätigkeit durch eine Staatsorganisation abzuhelfen, war für ihn ebenso wie für Locke ein Rückfall in den Feudalismus. Was aber sind Alternativen zur Markttätigkeit, die an Lockes revolutionärer Idee der Selbstvervollkommnung oder Emanzipation durch Arbeit festhalten?

\section{Alternativen zur Markttätigkeit?}

Welche Konsequenz hätte Locke für seinen Politikbegriff zu ziehen, wenn der Weg zum Besitzerwerb sich nicht als offen für alle erwiese? Besitzerwerb ist für Locke kein Selbstzweck, sondern das Mittel zu einer Vollkommenheit, die in rationalen Tätigkeiten besteht und heute auch mit Begriffen der Autonomie und Emanzipation beschrieben wird. Eigentum steht nicht nur unter der nach Locke leicht erfüllbaren Bedingung, dass ,there is enough, and as good left in common for others ${ }^{638}$, sondern auch unter der inhaltlichen Bedingung, dem ,use of the Industrious and Rational“" zu dienen. ${ }^{39}$ Die heutigen Marktverhältnisse aber fördern nicht mehr „the Industrious and Rational“. Die Konsequenz wäre daher für ihn gewesen, andere Wege zum Ziel der Vollkommenheit durch rationale Tätigkeit zu suchen.

Diese Konsequenz zog Marx. Überzeugt von der Unmöglichkeit, dass jeder Eigentum erwerben oder durch dessen Erwerb seine Vernunft betätigen könne, erklärte er:

„An die Stelle der alten bürgerlichen Gesellschaft mit ihren Klassen und Klassengegensätzen tritt eine Assoziation, worin die freie Entwicklung eines jeden die freie Entwicklung aller ist." ${ }^{40}$

Eine Assoziation, worin die freie Entwicklung eines jeden die freie Entwicklung aller ist, ist genau das, was Locke sich vom Staat versprach. Er würde Marx' Satz zustimmen, wäre bürgerlich nur durch feudal ersetzt. Die freie Entwicklung der Arbeit eines jeden in einem Marktsystem schien ihm, wie vielen späteren Ökonomen, notwendig die freie Entwicklung aller nach sich zu ziehen. Heute ist klar, dass der Markt zwar die Arbeitsproduktivität gewaltig gesteigert, aber gerade deswegen die Arbeit von immer mehr Individuen ökonomisch überflüssig gemacht hat. Unfreiwillige Arbeitslosigkeit und der Nichtgebrauch rationaler Fähigkeiten sind die Folgen. Die Arbeit, von der Locke die Vervollkommnung aller erwarte-

\footnotetext{
38 Ebd., § 27.

39 Ebd., $\$ 34$.

40 Marx u. Engels, Manifest der Kommunistischen Partei, in: MEW, Bd. 4, 482.
} 
te, war die Arbeit entweder eines Produzenten, der die materiellen Produktionsbedingungen besitzt und sein Produkt auf dem Markt verkauft, oder die Arbeit eines Besitzlosen, der seine Arbeitskraft verkauft. Heute dient sie nicht mehr der Vervollkommnung, weder der Natur noch des Menschen, wie die ökologischen Schäden, zunehmende Arbeitslosigkeit und ein Mangel an Möglichkeiten zur Eigeninitiative zeigen. Gibt es andere Wege, auf denen jeder zur Betätigung seiner rationalen Fähigkeiten kommen kann?

Zwei Wege sind für Locke und Marx gleichermaßen ausgeschlossen: der Einsatz des Staates zur Behebung von Marktproblemen und der Verzicht auf Austausch, der den Markt von vornherein überflüssig machen würde. Der Rückgriff auf den Staat, durch Arbeitsbeschaffungsprogramme oder Wirtschaftsplanung, ist für Locke wie für Marx ein Rückfall in den Feudalismus oder in Platons Staatsutopie. ${ }^{41}$ Nicht die Individuen würden durch ihre eigene person die Tätigkeit wählen, in der sie ihre rationalen Fähigkeiten entwickeln, sondern eine Obrigkeit, die ihnen Tätigkeiten zuweist. Die Gesellschaft bliebe gespalten in Entscheider, die den Arbeitsuchenden Arbeit zuweisen, und Befehlsempfänger; in „Arbeitgeber“ und „Arbeitnehmer“, wie es so treffend heißt. Sie wäre keine „Assoziation“ oder ,political society“, die sich aus den selbst gewählten rationalen Tätigkeiten der Individuen ergibt und nur die Aufgabe hat, nicht unbedingt Eigentum, wohl aber die Rechte der Individuen zu schützen. Nach Locke wie nach Marx kann es Verbesserung nur durch die Initiative jedes einzelnen Individuums geben. Dass der Markt gerade das zu erlauben scheint, machte ihn so attraktiv.

Der Verzicht auf Austausch ist ebenso wenig akzeptabel. Er wäre ein Rückfall in eine Autarkie, die den Individuen keine Entfaltung und keine Wahl ihrer individuellen Talente erlauben würde. Zur Entfaltung von Talenten gehört Kooperation, aber auch wechselseitige Kritik und Anregung, die nach der klassischen Ökonomie der Markt vermittelt. Wenn aber weder der Verzicht auf Austausch noch die Ersetzung des Marktes durch den Staat die heutigen Marktprobleme lösen kann, scheint eher eine Reform der Märkte als ihre Abschaffung angezeigt.

Auch nach Marx muss Austausch als Bedingung der Betätigung der individuellen Talente erhalten bleiben. Er beschreibt seine kommunistische Assoziation als einen Ort, „wo Jeder nicht einen ausschließlichen Kreis der Tätigkeit hat, sondern sich in jedem beliebigen Zweige ausbilden kann, die Gesellschaft die allgemeine Produktion regelt und mir eben dadurch möglich macht, heute dies, morgen jenes zu tun, morgens zu jagen, nachmittags zu fischen, abends Viehzucht zu treiben, nach dem Essen zu kritisieren, wie ich gerade Lust habe, ohne je Jäger, Fischer, Hirt oder Kritiker zu werden". 42

Der Markt ist hier durch „,die Gesellschaft“ ersetzt, die „,die allgemeine Produktion regelt“. Das scheint ein Vorgriff auf die Planwirtschaft der sozialistischen Staaten, die hinreichend gezeigt haben, wie unfähig sie zur Vervollkommnung der Menschen sind. Marx setzt jedoch der Produktionsregelung auch ein Ziel, dem die sozialistischen Planwirtschaften nicht gefolgt sind: Individuen zu ermöglichen, zu tun und zu lassen, wozu sie ,gerade Lust“ haben. Der flapsige Ausdruck, dem hochtrabenden Stil der kritisierten deutschen Ideologen entgegengesetzt, darf nicht als Spott auf das klassische Vervollkommnungsziel verstanden werden. Im Gegenteil geht es Marx und Engels gerade um dies Ziel. Allerdings vermeiden sie den Begriff der Vollkommenheit, der ihnen wie auch den meisten heutigen Zeitgenossen zu akademisch

41 Platons Staat folgt durchgehend dem Prinzip, dass jede Tätigkeit, auch die der Berufswahl, Gegenstand der Spezialisierung und eine Sache der dazu Fähigsten sein sollte, die in einer Staatshierarchie entscheiden.

42 Marx u. Engels, Die deutsche Ideologie, in: MEW, Bd. 3, 33. Francis Fukuyama (The End of History and the Last Man, New York 1992, 355) sagt: Diese „,vision [...] was [not] meant seriously“. 
und professoral ist. Sie gebrauchen vielmehr den Begriff der Emanzipation, an deren Ende eine Autonomie anzunehmen ist, die der traditionellen Vollkommenheit entspricht. Autonomie und Vollkommenheit schließen die rationale und voluntative Fähigkeit ein, die eignen Fähigkeiten zu erkennen und so zu betätigen, dass sowohl ich selbst als auch alle andern Vernunftwesen ihre Fähigkeiten betätigen können.

Eine Gesellschaft, die am Autonomieziel oder der Emanzipation aller orientiert ist, müsste sicherstellen, einerseits dass produziert wird, was jeder konsumieren will, anderseits dass jeder tun und lassen kann, wozu er Lust hat. Es ist sehr unwahrscheinlich, dass diese Doppelbedingung ohne Märkte erfüllbar ist. ${ }^{43}$ Lassen sich die Märkte wirklich nicht dem Autonomieziel unterordnen, wie Marx meinte? Ihre Unverzichtbarkeit scheint trotz aller Marktmiseren so überzeugend, dass wir fragen sollten, wie Marx sie für verzichtbar halten konnte. Schließlich hatte er ein scharfes Auge für gesellschaftliche Entwicklungen und Notwendigkeiten.

Die Erklärung scheint mir folgende. Er sah eine Entwicklung voraus, die ich Kommunismus in der Produktion nenne: die Verflechtung der Arbeiten der Individuen in einer Intensität, die die Zuordnung eines Wertanteils am Gesamtwert des gesellschaftlichen Produkts an ein Individuum unmöglich macht. Kommunismus in der Produktion schien ihm unvereinbar mit der heutigen Produktionsform, die von allen Produkten und Dienstleistungen Vermarktbarkeit oder Kommerzialisierung verlangt. Wegen dieser Unvereinbarkeit sagte er den Wegfall der Märkte voraus.

Wie ich nun zeigen möchte, haben sich Marx' Voraussage des Kommunismus in der Produktion und seine These der Unvereinbarkeit mit der heutigen Vermarktbarkeitsbedingung bestätigt. Aber sein Schluss auf den Wegfall der Märkte war irrig. Die Märkte überleben den Wegfall der Vermarktbarkeitsbedingung. Sie wechseln ihre Funktion. Sie lassen sich auf ihre ursprüngliche Funktion der Kontrolle der Produktion oder der Anpassung der Produktion an menschliche Bedürfnisse und Fähigkeiten, sogar an das Emanzipationsziel zurückführen.

Auch Locke sah, trotz seiner Betonung der Rolle der Individuen in der Produktion, dass die komplexe Arbeitsteilung der zeitgenössischen westeuropäischen Gesellschaften nicht zulässt, den Wert eines Produktes, wie eines Brotlaibs oder eines Hauses, einem Individuum gutzuschreiben. Selbst am Brot sind Bauer, Pflugschmied, Bergmann, Müller, Ofensetzer, Steinmetz, Bäcker beteiligt. Aber er hielt es für möglich, den Wertanteil der Arbeit jedes Mitproduzenten am Wert des Brots durch Berechnung seiner Arbeitszeit einschließlich seiner zur Erlernung der Arbeit notwendigen Ausbildungszeit zu berechnen. Marx teilte Lockes Auffassung für die Gesellschaft seiner Zeit, wie seine Aussagen zur Berechnung des Arbeitslohns zeigen. ${ }^{44}$ Jedoch hielt er eine Wertzuschreibung an die individuellen Produzenten für unmöglich, wenn die Zusammenarbeit eine gewisse Intensität erreicht.

Nehmen wir das Beispiel der Softwareproduktion. ${ }^{45}$ Bis in die 1970er Jahre erfolgte sie weitgehend im freien Austausch der Programmierer. Danach versuchten die Eigentümer der

43 Vielleicht gelingt es einem genialen Programmierer, den Markt durch eine Simulation zu ersetzen, der von allen Marktfehlern frei wäre. Ohne einen solchen virtuellen Markt müssen wir mit einem reformierten auskommen.

44 K. Marx, Kritik des Gothaer Programms, in: MEW, Bd. 19, 20: „Die individuelle Arbeitszeit des einzelnen Produzenten ist der von ihm gelieferte Teil des gesellschaftlichen Arbeitstags, sein Anteil daran. Er erhält von der Gesellschaft einen Schein, daß er soundso viel Arbeit geliefert (nach Abzug seiner Arbeit für die gemeinschaftlichen Fonds), und zieht mit diesem Schein aus dem gesellschaftlichen Vorrat von Konsumtionsmitteln soviel heraus, als gleich viel Arbeit kostet. Dasselbe Quantum Arbeit, das er der Gesellschaft in einer Form gegeben hat, erhält er in der andern zurück.“

45 Über sie informiert die Magisterarbeit von: J. Korb, Geschichte der freien Software, TU Berlin, 2001; vgl. auch P. E. Ceruzzi, A History of Modern Computing, Cambridge/Mass. 2000. 
Computer zunehmend, einzelne Beiträge zur Softwareentwicklung als geistiges Eigentum zu behandeln, den Programmierern geistige Eigentumsrechte zuzuschreiben und den Zugang zur Software durch Patente und Copyrights käuflich zu machen. Die Unterwerfung der Softwareproduktion unter Vermarktungsbedingungen stößt jedoch auf technische und mentale Widerstände. Es ist nicht einfach, Kopien neuer Programme zu verhindern, und der dazu nötige Aufwand scheint besser in der konstruktiven Programmentwicklung aufgehoben. Die freie, um Eigentumsrechte unbekümmerte Zusammenarbeit der Programmierer ist zu produktiv, als dass ihre Einschnürung durch Vermarktungsinteressen überzeugen könnte.

Die Erfahrung des Verlustes an Produktivität, aber auch am Spaß der Zusammenarbeit in der Programmentwicklung, brachte Richard Stallman dazu, die Privatisierung seiner Arbeit zu verweigern und ihr die Arbeitsform einer freien Softwareentwicklung entgegenzusetzen. Sie wurde als free software movement bekannt und bewies mit der Entwicklung des GNUProjekts und ihrer Beteiligung am Linuxprogramm ihre Produktivität. ${ }^{46}$

Die Entwicklung der Computerindustrie ist ein Musterfall der Entwicklung der Produktivkräfte in der heutigen Gesellschaft, der die wachsende Schwierigkeit in der Wertzumessung individueller Arbeiten beleuchtet. Ökonomen in ihrer Theorie und Arbeitgeber in ihrer Praxis sind sich heute einig, dass der Wert einer Arbeit oder eines Arbeitnehmers nicht an der Länge der Arbeitszeit und auch nicht an der Länge seiner Ausbildungszeit gemessen werden kann. Der Wert eines Arbeitnehmers hängt zu einem großen Teil an seiner Fähigkeit zu Teamwork, an seinem Talent, andre zu inspirieren und von ihnen inspiriert zu werden, Netzwerke mit Kollegen auch aus der Konkurrenz auf- und auszubauen, die in den vorangehenden Stationen seiner Ausbildung und Karriere gewonnenen Kenntnisse anzuwenden.

Wir können zwar argumentieren, dass der Wert eines Arbeitnehmers schon immer an Bedingungen hing, die nicht sein Verdienst waren. Schon immer waren Talent und Einsatz, Lockes rationality und industriousness, mitbestimmt durch Naturanlage und Erziehung, die nicht in der Kontrolle der Individuen liegt. Das hat nicht davon abhalten können, Individuen für ihre Handlungen verantwortlich zu machen. Aber was ich als Kommunismus in der Produktion beschreibe, ist von dieser grundsätzlichen Abhängigkeit der Leistung eines Individuums von Talent und Milieu unterschieden. In diesem Kommunismus kommt eine Leistung erst durch eine Kooperation von Individuen zu Stande, in der eine quantitative Aufteilung der Wertanteile auf die Kooperierenden unmöglich ist. Trotzdem Wertanteile am Gesamtprodukt den einzelnen Produzenten zuzuordnen, ist Willkür. Es geht an der Arbeitswirklichkeit vorbei und wird zunehmend absurd.

Das heißt nicht, dass das Individuum nichts ist als das „Ensemble der gesellschaftlichen Verhältnisse“, wie Marx es einmal beschrieben hat. ${ }^{47}$ Das Individuum mit seinen individuellen und unersetzlichen Eigenschaften wird eher wichtiger. Aber seine Leistung ist qualitativ; sie quantitativ zu bewerten, ist unangemessen. Zudem widerspricht es der Idee einer ökonomischen Ressourcenverteilung, die Leistung eines Individuums in einer Weise zu quantifizieren, in der es für Arbeit, die ihm Spaß macht und ohnehin höchste gesellschaftliche Anerkennung einbringt, das unglaublich Vielfache einer unangenehmen und verachteten Arbeit erhält.

Die Intensität der Arbeitsteilung, wie wir sie heute in der Computerindustrie beobachten, hat in vielen Bereichen einen Kommunismus in der Produktion geschaffen. Er wird gefördert durch die Möglichkeit, im Internet öffentliche Foren zu schaffen, an denen jeder Interessierte teilnehmen kann. Beispiele sind Wikipedia und WikiLeaks, die die Lexika und die Medien

46 Vgl. R. Stallman, Reevaluating Copyright. The Public Must Prevail, in: Oregon Law Review, 75 (1996); vgl. auch ders. u. a., GNU Make, Boston 2004.

47 K. Marx, Thesen über Feuerbach, These 6, in: MEW, Bd. 3, 6. 
revolutionieren. Ihr Erfolg beruht auf einer Kooperation, deren ökonomischer Wert nicht auf die Beiträger aufgeteilt werden kann. Sie widerlegen auch die traditionelle Annahme, dass Individuen nur durch Geldwert zu Leistungen motiviert werden. Der Spaß an der Sache und die öffentliche qualitative Anerkennung, die manche und nicht einmal alle Beiträger erhalten können, ist genug Motivation für Leistung.

Kommunismus in der Produktion ist unverträglich mit der Vermarktungsbedingung der kapitalistischen Produktionsform. Die Zuweisung von Wertanteilen des Endprodukts an einzelne Produzenten mit der Konsequenz der Einführung von geistigen Eigentumsrechten, Copyrights und Patenten für Schritte in einer Entwicklung von Programmen oder, in der Biotechnik, Gensequenzen, die von Gruppen von Technikern und Wissenschaftlern und nicht von Individuen vorangetrieben wird, hemmt die Entwicklung der Produktivität. Die Marktform der Produktion ist zu dem geworden, was Marx über sie behauptet hat: ein Hindernis, eine Fessel der Produktivkräfte. Sie hemmt nicht nur die Entwicklung der Technik. Sie verhindert auch die Kreativität, Kommunikativität, Produktivität und Autonomie der Menschen und verkrüppelt sie zu Tätern und Opfern der Vermarktung.

Aus diesen Tatsachen schloss Marx, die Märkte würden abgeschafft werden. Die Praxis der freien Softwarebewegung widerlegt diesen Schluss. Sie kommt nicht ohne Markt aus. Auch Linux und andre GNU-Produkte brauchen einen Markt, um ihre Anwender zu erreichen. ${ }^{48}$ Aber das heißt nicht notwendig, dass der Markt die freie Softwarebewegung umbringen wird. Der Markt, den freie und offene Softwareprodukte brauchen, hat nicht die Funktion, zu maximalem Gewinn anzureizen, sondern Fehler und Schwächen der Produkte zu entdecken. Dies ist die Funktion, die Ökonomen dem Markt grundsätzlich zusprechen: die Produktion den Konsumenten anzupassen. Diese Funktion wird immer leicht in eine Anpassung der Konsumenten an die Marktproduktion umschlagen können. Es wird an den Konsumenten liegen, das zu verhindern. Allerdings wird der Markt der freien Software vermutlich den übrigen Märkten angepasst werden, wenn nicht auch diese ihre ursprüngliche Funktion wiedergewinnen.

Wie lassen sich Märkte auf ihre Funktion zurückführen, die Produktion den Konsumenten anzupassen? Das ist die Frage, vor der heute Marxisten ebenso wie Locke-Liberale stehen, die am Ziel der Autonomie oder Emanzipation festhalten. Wer Lockes Utopie der Vervollkommnung jedes Individuums durch eigne Tätigkeit verteidigen will, muss einen Weg finden, den Kommunismus in der Produktion mit dem Markt verträglich zu machen. Vermutlich gibt es mehr als einen Weg zu diesem Ziel. Der Weg, auf den ich zum Abschluss verweisen möchte, ist der, ein bedingungslos ausgezahltes Grundeinkommen einzuführen.

Denken wir an zwei besonders verheerende Folgen der heutigen Märkte, an die zunehmende Arbeitslosigkeit infolge des Einsatzes arbeitssparender Maschinen und an die zunehmende Kommerzialisierung von Arbeitsbereichen wie Gesundheit, Erziehung, Sport, Nachrichten. Auf der einen Seite werden immer mehr Menschen auf dem traditionellen Gebiet der Produktion von Produktions- und Konsumgütern ökonomisch überflüssig; auf der anderen Seite werden Bereiche, deren Leistungen bisher nicht nach Marktprinzipien bezahlt, sondern durch „Honorare“ oder andere als qualitativ geltende „Gehälter“ vergütet wurden, Vermarktungsprinzipien unterworfen: Arzt, Lehrer, Journalist, Schriftsteller sollen ihre Arbeit als Dienstleistungen verstehen, die sie zum Gewinn eines privaten Unternehmens im Gesundheits-, Erziehungs- oder Kunstbereich zu verkaufen haben. Es geht nicht mehr darum, Menschen die beste Gesundheit, Erziehung, Information oder Kunst anzubieten, sondern aus diesen Berei-

48 Vgl. J. Bitzer u. P. J. H. Schröder (Hg.), The Economics of Open Source Software Development, Elsevier 2006. 
chen so viel Gewinn wie möglich zu schlagen. Solche dem Dienstleistungssektor zugeschlagenen Felder sollen den im Produktionsbereich überflüssig Gewordenen einen Arbeitsplatz verschaffen.

Diese Entwicklung ist eine weitere Anpassung der Menschen an ein Produktionssystem, in dem der Markt - ursprünglich ein Mittel, die Produktion menschlichen Fähigkeiten und Bedürfnissen anzupassen - in eine Institution umgeschlagen ist, die die menschlichen Fähigkeiten und Bedürfnisse dem Profitziel anpasst. Die Entwicklung ist ein weiteres Paradox in der langen Geschichte von Paradoxien, in der die Märkte ihre heutige Stellung erobert haben. Die Automatisierung der Produktion ist eine offensichtliche Gelegenheit, eine radikale Verkürzung der durchschnittlichen Arbeitszeit durchzusetzen. Stattdessen macht der Druck der Arbeitslosigkeit die Menschen bereit, zusätzliche Arbeit auf sich zu nehmen, dadurch die Arbeitslosigkeit zu steigern und die Umwandlung bisher nicht profitorientierter Arbeitsbereiche in profitorientierte hinzunehmen und an das Argument zu glauben, dadurch würden zusätzliche Arbeitsplätze geschaffen. Wie kann diese Entwicklung gestoppt werden?

Es gibt einen weiteren Aspekt der von Locke empfohlenen Marktgesellschaft, den Locke noch nicht bedenken konnte. Welchem Beruf jemand nachgeht, sollte nach Lockes Idee der gleichen Freiheit und Selbstbestimmung von jedem selbst gewählt werden. Tatsächlich aber wird der Beruf den meisten Individuen vom Arbeitsmarkt diktiert, was der Idee der Selbstbestimmung Hohn spricht. Kann diese Idee überhaupt noch eine Rolle spielen?

Eine Möglichkeit, die Übermacht der Märkte und besonders des Arbeitsmarkts zu verringern, scheint die Organisierung der Bereiche der Gesundheit, Erziehung, Information, Kunst durch den Staat. Schulen und Universitäten, Krankenhäuser und Fernsehanstalten sind in der Tat noch heute großteils staatlich organisiert oder unter staatlicher Aufsicht und sind dabei nicht immer schlecht gefahren. Aber ihre staatliche Organisation widerspricht der Idee der Autonomie und Emanzipation sowohl von Locke wie Marx. Sie hält, wie gesagt, an der Teilung von Befehlgebern und Befehlnehmern fest. Um sie zu vermeiden, müssen die Individuen selbst entscheiden können, wie sie ihre Fähigkeiten gebrauchen wollen. Sie müssen von Arbeitsmarkt und Arbeitsamt unabhängig sein. Diese Unabhängigkeit erreichen sie, wenn jeder ein bedingungslos ausgezahltes Grundeinkommen erhält, eine Summe, die für eine bescheidene Lebensführung ausreicht. ${ }^{49}$

Auf den ersten Blick verstärkt ein Grundeinkommen die Macht des Staats, gegen die ich mich gerade gewandt habe. Das ist jedoch nur der Fall, wenn der Staat das zum Grundeinkommen nötige Geld eintreibt und verteilt. Das nötige Geld aber kann in jedem Fall nur von den Unternehmen kommen. Der Druck, der Unternehmen zur Einsicht bringt, dass es zu ihrem eignen Vorteil ist, durch ein Grundeinkommen zur Verbesserung der Gesellschaften beizutragen, muss nicht vom Staat kommen. Ein Streik etwa der Informatiker oder der Mediziner für die Einführung des Grundeinkommens würde schnell den gewünschten Erfolg bringen. Moderne Gesellschaften sind durch den zunehmenden Kommunismus in der Produktion so empfindlich gegen Streiks geworden, dass Streiks zu einer wirksameren Waffe geworden sind als je zuvor. ${ }^{50}$

49 Vgl. W. Götz u. A. Goehler, 1000 Euro für jeden. Freiheit, Gleichheit, Grundeinkommen, Berlin 2010.

50 Vgl. R. Ramtin, Capitalism and Automation, London 1991, 15: „The advance of automation, rather than resolving the ,problem' of class conflict, actually transforms it by transforming the struggle from its hitherto restricted and, as it were, domesticated form of, to a large extent, isolated, localized battles over wages and conditions of labour into more immediately and directly political struggles $[\ldots]$ as living labour is removed from the point of production, the antagonistic relations in production are actually transferred from the site of production, from within the walls of the various factories and 
Staatsunabhängige Organisationen wären auch vertrauenswürdiger in der Eintreibung und Verteilung der Gelder. Daher kann die Einführung des bedingungslosen Grundeinkommens zum Abbau der Staatsmacht und zum Aufbau staatsunabhängiger öffentlicher Institutionen beitragen.

Drei weitere wichtige Einwände werden gegen das Grundeinkommen erhoben: Es sei nicht finanzierbar, nicht gerecht, nicht emanzipatorisch. Hier nur kurze Kommentare.

Der Finanzierbarkeitseinwand ist am leichtesten auszuräumen, weil auch etablierte Ökonomen das Grundeinkommen für finanzierbar halten. ${ }^{51}$ Grundsätzlich kann man erwarten, dass dieselbe Produktivität, die Arbeitslosigkeit erzeugt, auch die Güter produziert, die die arbeitslos Gewordenen zum Lebensunterhalt brauchen.

Der Gerechtigkeitseinwand wiegt schwerer, da das Grundeinkommen Individuen erlaubt, von Werten zu leben, die andere geschaffen haben. Ist es kein Unrecht der Faulen an den Fleißigen? Dieser Einwand übersieht, dass die Kategorien von Faulheit und Fleiß ihre Rolle verloren haben. Früher waren Arbeitskräfte knapp und Arbeitsplätze im Überfluss vorhanden. Nach dem Wertgesetz, dass Knappheit den Wert einer Sache bestimmt, sind Arbeitsplätze wertvoll und ihr Besitz ein Privileg geworden. Untätigkeit wurde zu Recht mit Verachtung gestraft, solange der Wohlstand einer Gesellschaft an der Zahl der Produzenten hing. Heute ist es die Aufgabe verantwortlicher Politik, klar zu machen, dass Arbeitslosigkeit oder die Nichtvermittelbarkeit bestimmter Arbeiten auf dem Markt nicht bestraft werden darf.

Am schwersten wiegt der Einwand, ein Grundeinkommen diene nicht der Emanzipation. Es mag Individuen ermöglichen, zu tun und zu lassen, wozu sie grad Lust haben. Aber wird es sie emanzipieren, autonom machen oder, wie man traditioneller gesagt hat, vervollkommnen? Werden sie nicht noch passiver, noch weniger Eigeninitiative zeigen, noch mehr den Sinn ihrer Existenz im Konsumieren suchen?

Ein bescheidenes Grundeinkommen macht es schwer, Sinn im Konsum zu finden. Es gibt lediglich Existenzsicherheit und erlaubt damit, langfristigen Zielen zu folgen. Nicht alle werden ihre Chance nutzen, sich in einer selbst gewählten Tätigkeit zu entwickeln. Aber einige werden sich in Bereichen engagieren, die sie interessieren, auf Partner mit ähnlichen Interessen stoßen und neue, von Marktzwängen unbehelligte Tätigkeitsfelder im Umweltschutz und anderen öffentlichen Angelegenheiten, den Medien, der Erziehung, der Technologie, dem Sport, der Kunst, der Wissenschaft entwickeln.

Viele werden aber auch eine Erwerbsarbeit wählen, um ihr bescheidenes Grundeinkommen zu erhöhen. Firmen müssten zwar für die unangenehmsten Arbeiten, die heute oft besonders schlecht bezahlt sind, sehr viel mehr bieten und für Direktionsposten sehr viel weniger. Aber das würde nur dem allgemeinen Gerechtigkeitsempfinden entsprechen. Durch die Höhe des Grundeinkommens könnte man auf dem Arbeitsmarkt Angebot und Nachfrage ausgleichen und einerseits die Arbeitslosigkeit ohne Schaden für die Erwerbsarbeit auf Null senken, anderseits den Rückgang der Nachfrage nach Erwerbsarbeit unter eine zur ausreichenden Versorgung notwendige Größe verhindern.

Die Einführung eines Grundeinkommens würde nicht von heute auf morgen die Gesellschaft revolutionieren, langfristig aber der Locke und Marx gemeinsamen Utopie einer emanzipierten Gesellschaft, einer Assoziation autonomer Individuen nahe kommen. Jeder könnte

offices, to the outside. “ Zu diesem Öffentlich- und Politischwerden des durch die Vermarktungsform behinderten Produktionspotenzials tragen natürlich auch die modernen Kommunikationsformen des Internet und Handy bei.

51 Vgl. etwa T. Straubhaar, Ein Grundeinkommen für alle, HWWI (Hamburgisches WeltWirtschaftsInstitut) Standpunkt, Februar 2010. 
die Tätigkeit wählen, die seinen Talenten und Interessen entspricht; jeder bliebe in seinem Erfolg aber auch vom Interesse abhängig, das er bei anderen für seine Tätigkeit wecken muss; entweder auf dem Arbeitsmarkt oder in den informellen Gruppen, die marktunabhängig um die selbst gewählten Tätigkeiten entstehen. Nicht zuletzt würde die Staatsbürokratie überflüssig und der Staat auf die Aufgabe der Durchsetzung von Gerechtigkeit beschränkt werden.

Prof. Dr. Ulrich Steinvorth, Bilkent University, Department of Philosophy, 06800 Ankara, Türkei

\begin{abstract}
In the first part of the paper, I describe the role labour, under conditions no longer existent, played for the perfection of individuals in Locke's political philosophy. Or to use a more up-to-date term, I describe labour's emancipatory role. In the second part, I examine the conditions for realizing Locke's aim of a society of autonomous individuals with a minimum of state power and market coercion, starting from a society whose members are becoming increasingly economically superabundant.
\end{abstract}

